\title{
Impact of Short-Term Variations in the Generation Output of Geographically Dispersed PV Systems
}

\author{
Insu Kim \\ Alabama A\&M \\ University \\ kobekim@,gmail.com
}

\author{
Miroslav Begovic \\ Texas A\&M \\ University \\ begovic@,tamu.edu
}

\author{
Branislav \\ Vidakovic \\ Georgia Tech \\ brani@gatech.edu
}

\author{
Petar Djuric \\ Stony Brook \\ University \\ pmdjuric@gmail.com
}

\author{
Vladimir Jeremic \\ Security Learning \\ Services, IBM \\ vjeremy@gmail.com
}

\begin{abstract}
When viewed in hourly intervals, a solar photovoltaic $(P V)$ system appears to have a more stable output than usual. However, there are short-term rapid variations in its generation output that result from transient cloudiness and weather disturbances in the atmosphere. By using Monte Carlo simulations applied to a Markov model, this study demonstrates the shortterm intermittency of the transient weather conditions and estimates the generation of geographically dispersed PV systems with a capacity of ten percent of peak demand of a statewide grid in one-minute intervals. This study found that geographically distributed PV systems evaluated in one-minute intervals could cope with peaks of a statewide power grid because of the smoothing effect caused by the geographical spread. The purpose of the exercise is to create a framework for integration and optimization of multiple generation sources in order to meet the uncertainty of the fast changing PV output under certain weather conditions.
\end{abstract}

\section{Introduction}

As renewable energy becomes one of the most popular new addition to the energy mix of many utilities around the world, trends in planning of generation are shifting focus from peak load to net load and system energy [1]. The pressure of variability of renewable generation resources is creating additional complexity on the non-renewable portion of the generation portfolio, with a consequence of a net increase of operating costs. Solutions to the overall problem of net load are found (in smaller systems) in combination of energy storage, demand response (or demand side management), and maintaining a sufficient amount of spinning reserve in the energy mix to accommodate the worst case scenarios of renewable generation variability. An analysis of the National Renewable Energy Laboratory (NREL) of large scale renewable penetration and area-to-area variability in hour and sub-hour time frames suggests that geographic diversity of the wind and solar plants ensures that the extreme changes in generation never occur (tested on a limited data set from 2006) [2]. Simple rules have been proposed in that report to accommodate for sub-hourly renewable generation variability.

In this paper, we are addressing two approaches to studying the impact of renewable generation variability on grid operation and planning. The first approach attempts to model the short-time resolution (sub-hour to minute level) of individual and geographically dispersed solar PV systems. A Markov weather model is built from measurement data and its performance is evaluated by stochastic simulations.

To quantify differences between production patterns, we propose two complementary approaches. The first is based on multiresoluton covariances that utilize nondecimated wavelet transforms [17] of the production patterns and that enable scale-dependent assessment of similarity/dissimilarity. The choice of nondecimated (stationary, maximum overlap) transforms is motivated by the fact that such transforms allow for arbitrary-sized inputs and preserve the time-locality across the multiresolution levels. As a result, such wavelet-defined covariances are capable of assessing similarity at various time scales/levels simultaneously. In addition, with such transforms, the monitoring of level-covariances or levelcorrelations can be carried out in real time.

The second approach involves analysis of normalized production patterns that can be thought of as probability densities. Distance measures between densities quantify their shape differences and capacity for stable outputs. We consider popular measures such as Hellinger, KullbackLeibler, and Divergence distances, [19], and demonstrate that they increase in magnitude when the spatial distance between the stations increases.

\section{Problem Statement}

The objective of this study is to (a) synthesize the shortterm, particularly in minute resolution, variation in the 
generation output of PV systems geographically dispersed across representative sites of a statewide grid and (b) assess the effect of PV systems on the operation of a statewide grid. For this purpose, this study performs a Markov chain Monte Carlo (MCMC) simulation because the output of PV systems shows a Markovian dependence. In other words, their output depends only on the current state and not on the previous history. We emphasize that generalizations are possible and readily implementable. The Markov chain can be of $k$ th order, meaning that in addition to the current state, $(k-1)$ previous states would determine the future state.

With some expense in computational complexity, we can also define time-dependent transition probabilities and simulate the system via continuous time Markov chain transitions.

\section{Markov Chain Monte Carlo}

For $n$ states, a transition probability of the first-order Markov chain process can be formulated through the following equation [1]:

$$
P[X(t+1)=j \mid X(t)=i)=P_{i j}, i, j=1, \cdots, n
$$

All the transition probabilities, which are constant in time, can be represented by the $n \times n$ matrix:

$$
P=\left[\begin{array}{ccc}
p_{11} & \cdots & p_{1 n} \\
\vdots & \ddots & \vdots \\
p_{n 1} & \cdots & p_{n n}
\end{array}\right]
$$

where $n$ is the total number of states. Since the sum of all transition probabilities from a single state to any other state is equal to one, the sum of each row in (2) is unity. That is,

$$
\sum_{j=1}^{n} p_{i j}=1, \quad i=1,2, \cdots, n .
$$

The cumulative distribution function of state $m$ can be expressed:

$$
F_{m j}=\sum_{k=1}^{m} p_{m k}, \quad m, j=1,2, \cdots, n .
$$

After determining the transition probability matrix, we propose to perform a Monte Carlo simulation of the Markov chain model. Uniformly distributed random sampling of a transition from a current state to the next state is proposed in [2-5]. This study applies this random sampling to the proposed MCMC simulation that synthesizes rapid variations in the generation output of geographically distributed PV systems in minute intervals. If a uniformly generated random number, $U$, falls within the boundaries of the cumulative probabilities of states $(j-1)$ and $j$, this study selects state $j$ as the next state from current state $I$, i.e.,

$$
j=k \quad \text { if } F_{i, k-1}<U \leq F_{i, k}
$$

where $U \sim \operatorname{Unif}[0,1]$, and $F_{i, 0}=0$, and $F_{i, n}=1$.

\section{Case Study}

\section{A. Large PV Systems Connected to a Statewide Grid}

As an example, we use a simulated case study of a set of 19 solar PV plants distributed across Georgia, USA (Figure 1). To synthesize the short-term variation in the generation output of large PV systems connected to a statewide grid, this study estimates their output in hourly and minute-byminute resolutions. In hourly resolution, it is assumed that all PV systems are geographically dispersed across the representative 19 sites across Georgia and have the capacity equal to $10 \%$ of the total peak demand, or 17.152 $\mathrm{GW}$ in 2010 [6]. The disposition of the cluster of solar farms is depicted in Figure 1. To produce 10\% of the peak demand, multiple PV systems oriented at an azimuth of $180^{\circ}$ (facing south) and a tilt angle of $30^{\circ}$ (approximate geographic latitude) are installed in multiple locations. The capacity of the PV systems in each site is chosen to be proportional to the population of each site in 2011 [7].

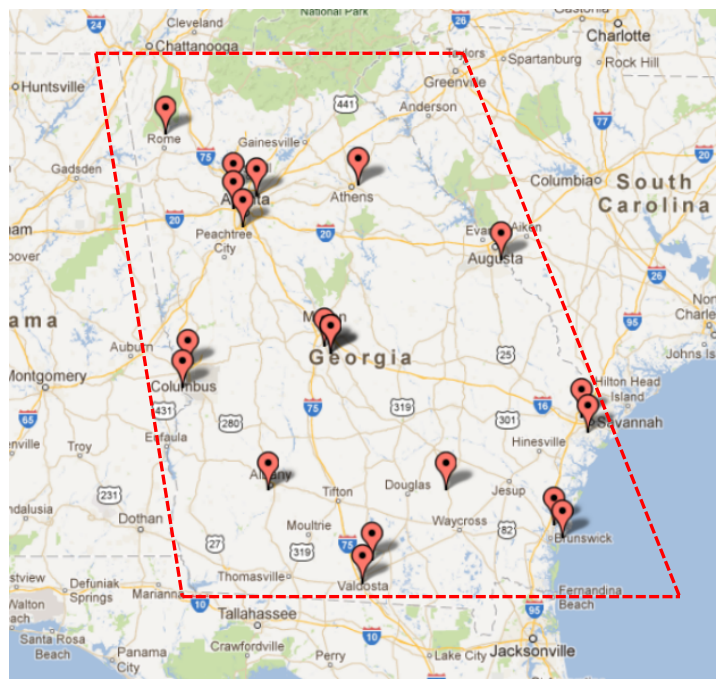

Figure 1. PV systems dispersed throughout 19 sites across Georgia [7].

For example, since 77,683 people populate in the Albany area in Georgia in 2011 [8], which is $5.23 \%$ of the total number of population of the 19 sites, the PV systems installed in the Albany area are assumed to be $5.25 \%$ of the total capacity of the PV systems dispersed through the 19 sites of the state. This is roughly consistent with the 
assumption that certain fraction of the homeowners would choose to install PV systems on the roofs of their residences.

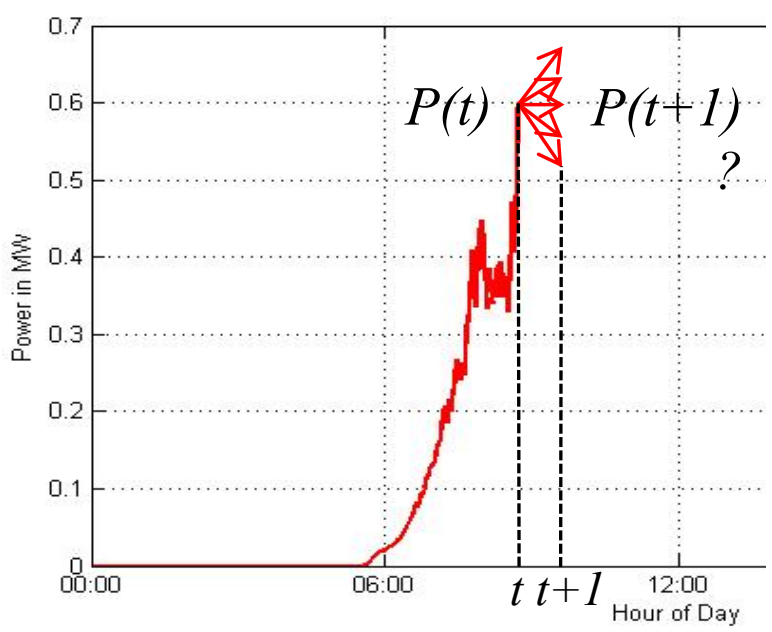

Figure 2. Illustration of the Markov chain model of transition probabilities of changes in solar radiation. Resolution is determined by the size of ranges of the final transition state.

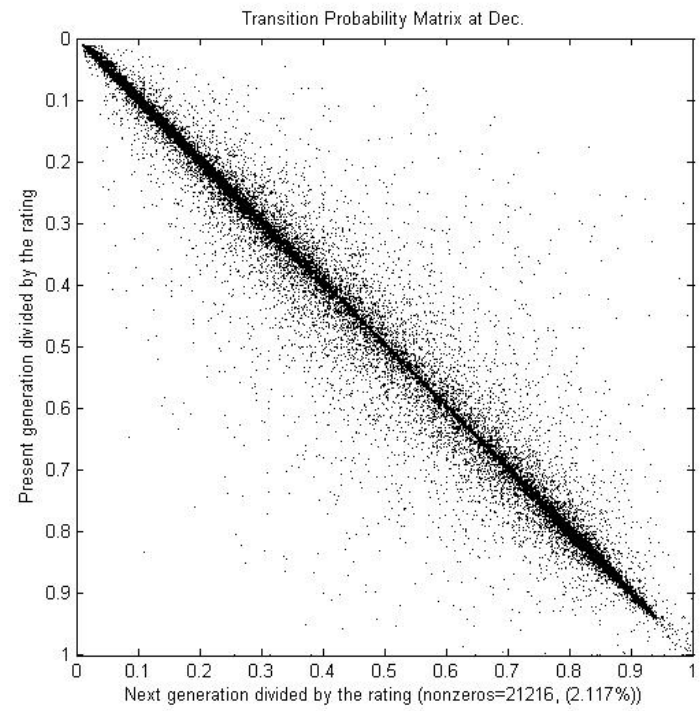

Figure 3. Representation [13] of the transition probability matrix of the Markov chain model of solar intensity transitions in resolution of $0.1 \%$. Only $2.117 \%$ of the transition coefficients are non-zero.

\section{B. Short-Term Intermittency of the PV System}

This study collected hourly solar data to estimate the generation output of the PV systems installed on the 19 sites of the state. Unfortunately, solar data in minute intervals for the state are not available from the current national solar radiation database [9]. Rapid variations in short-term, typically minute-averaging resolution, PV generation result from transient cloudiness and weather disturbances in the atmosphere [10]. Since current PV generation affected by transient cloudiness depends only on previous generation, rapid variations in PV generation can be modeled by optimized Markov chain methods [2, 4, 11]. Thus, to model the short-term intermittency of the transient cloudiness of the state, this study uses a Markov chain Monte Carlo (MCMC) simulation [7, 10, 12].

\section{Markov Chain Monte Carlo Simulation for PV Systems Dispersed across Georgia}

To calculate the transition probability matrix required as input data for a MCMC simulation, we collect a sample of actual solar data in minute-by-minute resolution from nine test sites located in Colorado, Arizona, New Mexico, and Utah from 2008 to 2012 (see Figure 4) [13], and estimate their PV outputs in minute intervals, using PV_LIB [14]. For example, on August 23, if a 1.4 MW PV system (with Canadian Solar CS5P-220M modules and a 1.4 MW Siemens inverter) were installed in each nine site, they could show short-term variations in their generation output, as shown in Figure 5. Figure 6 presents the synthesized generation output of the PV systems by summing their generation output. This study calculated the transition probability matrix of all nine sites during each month from 2008 to 2012 and selected a transition probability matrix calculated from the Milford area in Utah since it shows an annual output that most closely resembles that of the state.

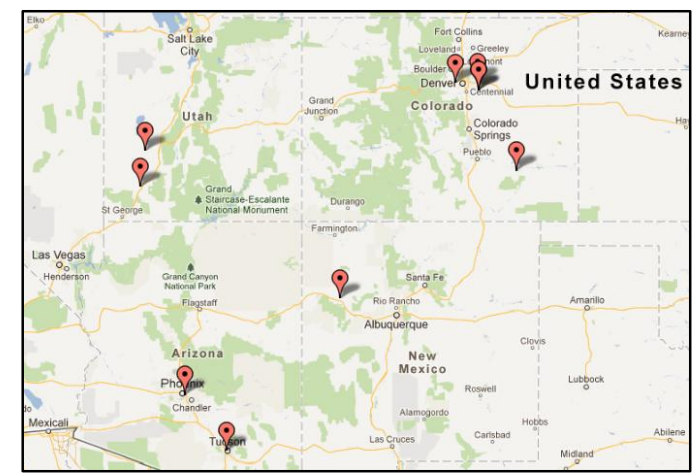

Figure 4. Solar irradiation data collected from the nine sites of US Southwest in minute-by-minute resolution.

As input data to the MCMC simulation and the transition probability matrix calculated from the generation output of PV systems in the Milford area, we use hourly solar data collected from the 19 sites of the state in the TMY3 format [9]. Figure 7 shows the generation output of a 1.4 MW single PV system that consists of Canadian Solar modules (CS5P-220M) and a 1.4 MW Siemens inverter (SINVERT PVS1401 UL) on July 4. The red curves in the figure indicate the generation output of the PV system in each site, discretized, averaged and presented in hourly intervals. The black curves illustrate the minute-by-minute 
synthesized generation outputs of the PV systems. They were obtained by the MCMC simulation method.
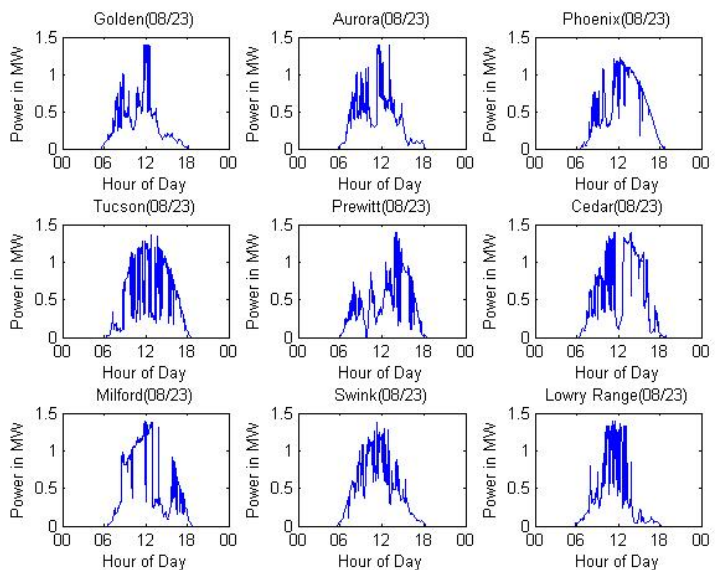

Figure 5. Generation output of a 1.4 MW PV system installed in the nine sites of US Southwest on August 23 [13]. $8 / 23 / 2012$

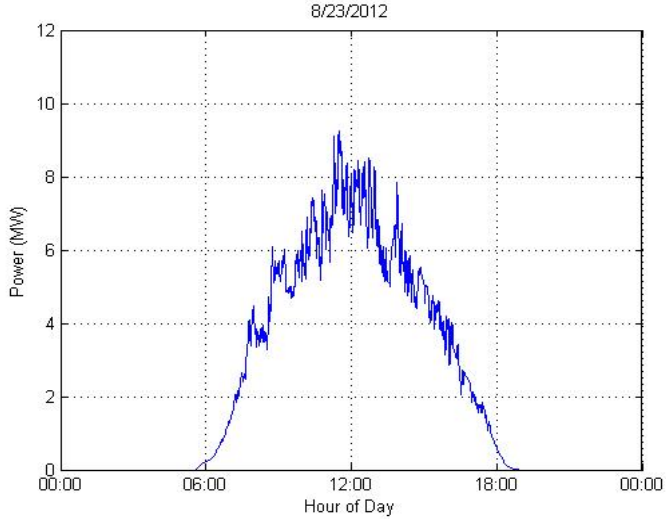

Figure 6. The generation output of total PV systems showing rapid variations on August 23.

As an example, let the current PV generation at hour $h$ be $P(h)$ PU, which is estimated from hourly solar data. To estimate the PV generation output in the next minute, this study generates a uniformly distributed random number, $u$, determines the boundaries of the cumulative probabilities of the transition probability matrix that $u$ belongs to, and estimates the generation output of the next state as the generation output corresponding to $u$, or $P(h)+p$ PU. That is, $P(h+1 / 60)=P(h)+p$ PU. The results in Figure 8 show that the total generation of the PV systems geographically dispersed across Georgia that produce $10 \%$ of the peak demand in minute resolution exhibit rapid short-term intermittency, which is comparable to that in Figure 6. Figure 7 and Figure 8 indicate that the rapid variations in the generation output of geographically dispersed PV systems are reduced because of the geographical spread, which is referred to as smoothing effect. Therefore, it seems that geographically distributed PV systems operating in oneminute intervals deal effectively with peaks of the statewide power grid. The reduction of the rapid variations in the combined PV system output is approximately an order of magnitude smaller than the variations of the individual plants that comprise the system (Figure 8).
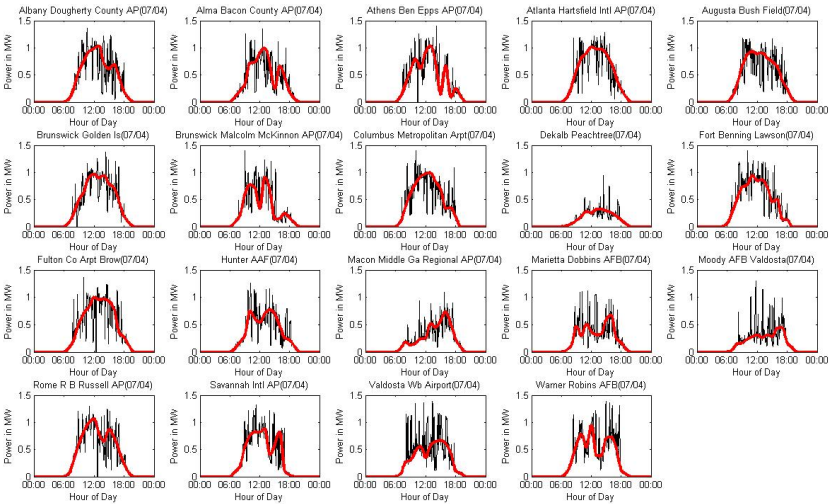

Figure 7. Generation output of a 1.4 MW PV system in the 19 sites of the state of Georgia. Red curves represent hourly data, while black curves represent minute-resolution synthesized solar data [13].

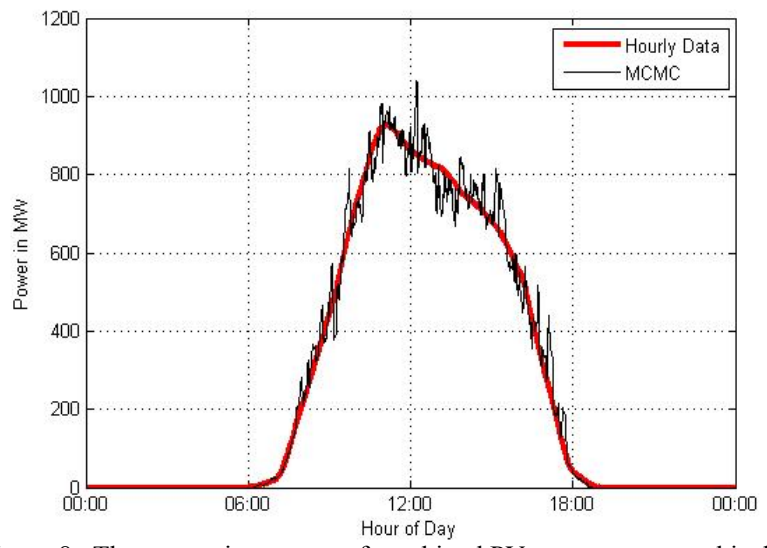

Figure 8. The generation output of combined PV systems geographically dispersed across the 19 sites, on September 27, 2010 shows a significantly smaller variation of the solar output on a transiently cloudy day.

\section{Assessment of Dissimilarities in Daily Short Term Production Patterns}

In the second approach to studying production patterns, we focus on quantifying the effects of randomness in renewable solar generation. We are interested in measuring the variability of production in a spatially distributed cluster of plants (units). It is expected that, when the production outputs are averaged over the number of units, the production pattern becomes less variable compared to the output from an individual unit. Also, the measure of similarity of production patterns depends, as expected, on the distance between the units. In this section we quantify these statements and propose a methodology 
for a formal assessment of measures of similarity of production patterns and ultimately a measure of system's production uniformity (and ability to forecast its production).

We start with an example. Consider a unit located in a cluster of stations in western Texas (Figure 9). Among 41 stations in the cluster for our illustration, we select the station located at $(32.55 \mathrm{~N}, 102.75 \mathrm{~W})$, with capacity of 118 MW. For a selected day, June 27, 1966, the production of this unit is presented in Figure 10 as red curve, the cumulative production of all 41 units from this cluster for the same day is presented in black, and the summary production of all cluster units, over the year period is presented in green. For comparison purposes, the outputs are normalized (as probability distributions), so the area below each curve is equal to 1. It is apparent that the variability of solar PV generation decreases with averaging with respect to cluster units and ultimately with averaging with respect to time.

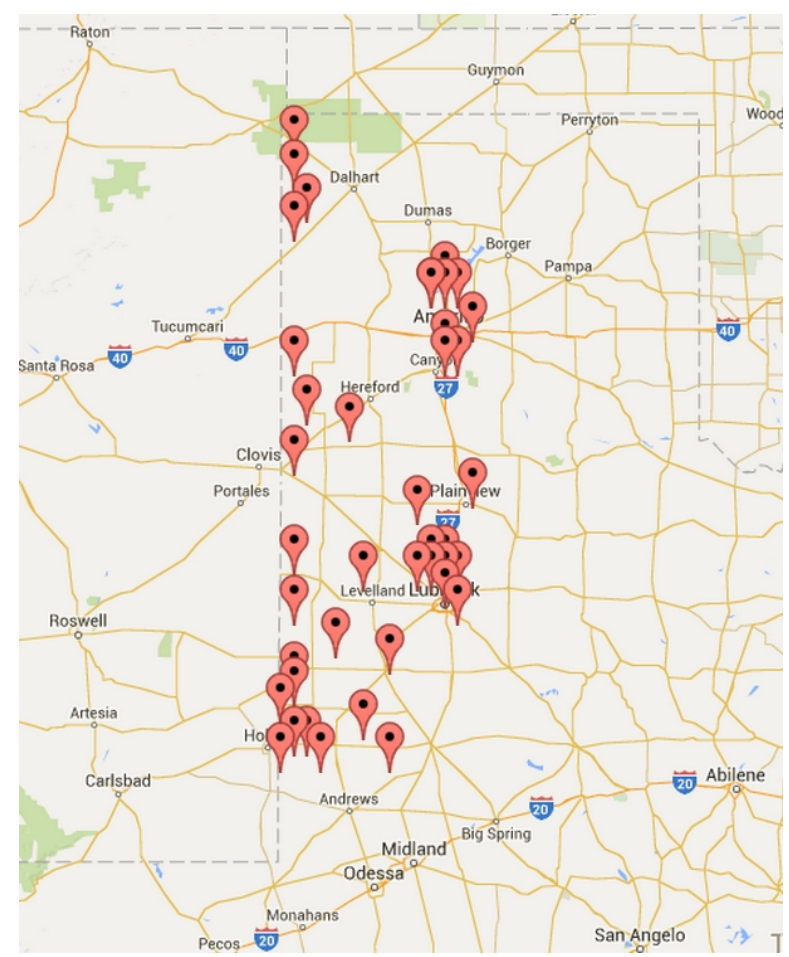

Figure 9. Locations of the hypothetical cluster of 41 solar farms in Western Texas

To facilitate power stability of the production, it is desirable that different units exhibit dissimilar compensating concurrent production patterns. We expect to find that the dissimilarity increases with the distance between the units. To this end, we define dissimilarity measures in two ways: (1) as absence of strong positive correlation among production patterns, and (2) as a distance between the normalized production patterns, in the spirit of distances between probability measures.

For global correlation between two patterns, we use the Spearman's correlation coefficient since it does not depend on the inherent Gaussian assumptions required by the standard Pearson's correlation coefficient.

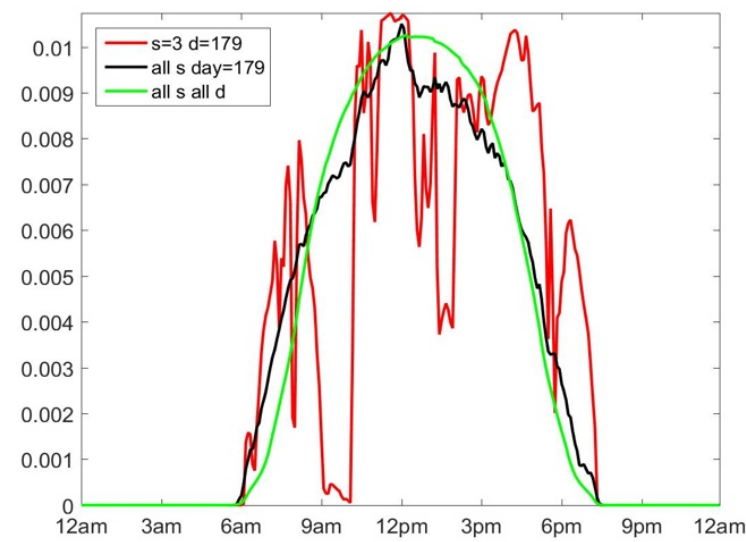

Figure 10: In red, daily production by the station \#3 during June 27, 1966. The normalized cumulative production on the same day by 41 units from a cluster located nearby (within about 200 miles) is presented in black. The green density shows normalized cumulative yearly production for the cluster. As expected, the smoothness increases when averaging by the units and time.

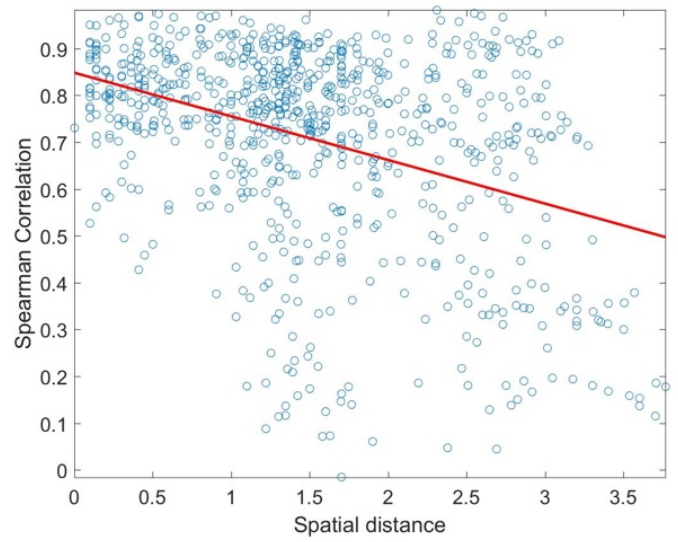

Figure 11: Spearman correlations between all pairs of units in the western cluster plotted against distance (the reference unit distance is 1 degree, which is approximately $111 \mathrm{~km}$ ). Note the negative slope.

By looking at the regression of the correlation coefficient on the distance between the units we find a statistically significant negative slope, suggesting that faraway patterns are more dissimilar and potentially more compensating. Figure 11 shows the Spearman correlation between all possible pairs in the cluster plotted against the distance between pair's units. Although the scatterplot is noisy, the negatively-sloped regression has significant slope. The null hypothesis that the slope is null was rejected with p-value smaller than $10^{-3}$. 
We found that Pearson's and Kendall's correlations behave in the same way, yielding significant negative slope in the best linear fit of correlations against the corresponding distances.

Although correlations, as measures of similarity of patterns, are time honored and well understood, they could be misleading for some pattern configurations. The patterns could be functionally dissimilar and compensating, yet the correlation would measure as high. This is because correlation is assessed at the sampling resolution. Potentially more informative measure will be the wavelet cospectra, or its normalized version, wavelet-based correlation. The construction of wavelet cospectra is simple: the two patterns are decomposed to the wavelet domain as a hierarchy of multiscale parts. The coefficients from the corresponding parts are compared and their covariance is assessed. This produces a measure of similarity that is resolutions/scale sensitive. As such, this measure can capture similarities/dissimilarities at time resolutions that are dyadic multiples of the data acquisition sampling rate. As an example, imagine two highly correlated patterns with a noise added to each. Depending on the level of the noise, standard correlation measures could miss the present coarse-level correlation. However, in the multiscale approach, the similarity will be captured at the resolution scale in which the similar patterns "live."

Figure 12 provides an example of normalized wavelet cospectra. To illustrate wavelet cospectra, July 24, 1996 outputs from four stations in the Western Texas cluster were selected. One station was acting as a baseline, and the other three were at distances 1.14, 2.4, and $3.7 \mathrm{deg}$. units, respectively, from it. We present normalized wavelet cospectra in Figure 12. Note that the close units 1 and 2 are similar at all scales. The units 3 and 4 are dissimilar with 1 at small scales 10'- 80', but become similar at the scale of 160' and higher. Operationally, the signals 1 and $(3,4)$ are compensating each other at time scales smaller than approximately 2 hours, but are synchronized at larger time scales.

To measure dissimilarity among production patterns, an alternative approach has a potential. Since the daily production patterns, considered as functions, are nonnegative and have a bounded domain, it is easy to normalize them and consider as probability densities. The measures of distances among probability measures is a well understood and well researched topic among probabilists. Since the supports of the two densities to be compared are approximately equal, the difference among them depends only on their shape.

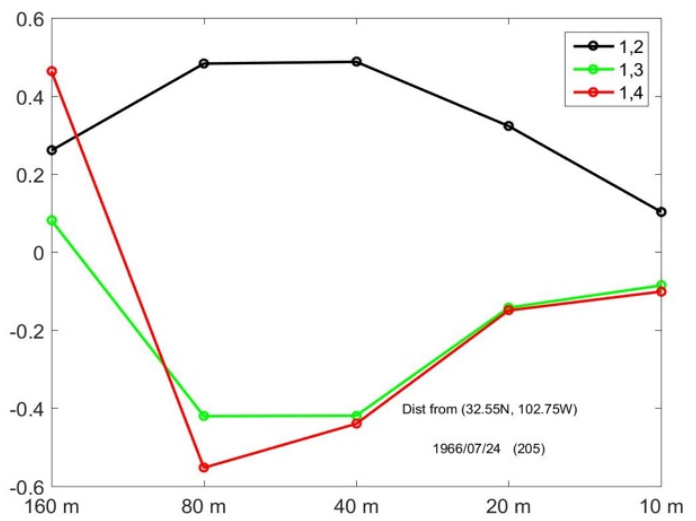

Figure 12: Wavelet-based correlation between units $(1,2)$ in black, $(1,3)$ in green, and $(1,4)$ in red. The distances between the pairs are distance $1.14,2.4$, and $3.7 \mathrm{deg}$, respectively. Note that close units $(1,2)$ are well correlated at most of the scales, while more distant pairs $(1,2)$ and $(1,4)$ are negatively correlated at scales up to $80^{\prime}$.

When the difference between densities is large, it is expected the station outputs will be compensating each other. We consider three standard measures of difference between densities: Hellinger, Kullback-Leibler, and Divergence distances. For two densities $f$ and $g$, the distances are defined respectively as

$$
\begin{gathered}
d_{H}=\frac{1}{2} \int(\sqrt{f(x)}-\sqrt{g}(x))^{2} d x, \\
d_{K L}=\int f(x) \cdot \log \frac{g(x)}{f(x)} d x, \\
d_{D}=2 \int \frac{(f(x)-g(x))^{2}}{f(x)+g(x)} d x .
\end{gathered}
$$

Except the Kulback-Leibler distance, which is not symmetric in its arguments, the other two distances are well calibrated and satisfy properties of symmetry and the triangle inequality. Intuitively, small distances would be linked to the equi-behavior of production patterns. In the presence of variability of patterns, this would indicate synchronization. On the other hand, large distances in normalized production densities will be linked with different shapes that are complementary in their convolution.

As expected, we found that each of the three distances between normalized production patterns increase with the distance between the units. To illustrate the performance of this collection of measures, we selected the Hellinger distance. Figure 13 shows the Hellinger distance between station \#3 and other stations from the cluster as a scatterplot against the respective spatial distance. Note, as expected, that the dissimilarity between patterns increases as the distance between the units increases, and this increase, statistically assessed, was significant. We found that the 
Kulback-Leibler and Divergence distances behave similarly, although the practical assessment of the Kiulback-Leibler distance could cause numerical instabilities (because of the logarithm of the ratio of the functions is obtained empirically).

In conclusion, both, the multiscale covariance measures and distances between probability measures showed potential in assessing similarity of production patterns. Such measures encourage further exploration and will play a pivotal role in the design of robust systems of geographically diversified plants. There, the smoothing compensation of production patterns among the units is maximized, thus reducing the cost of additional measures, which would be needed to accommodate the rapid variations of the solar plant outputs, especially at higher penetration levels.

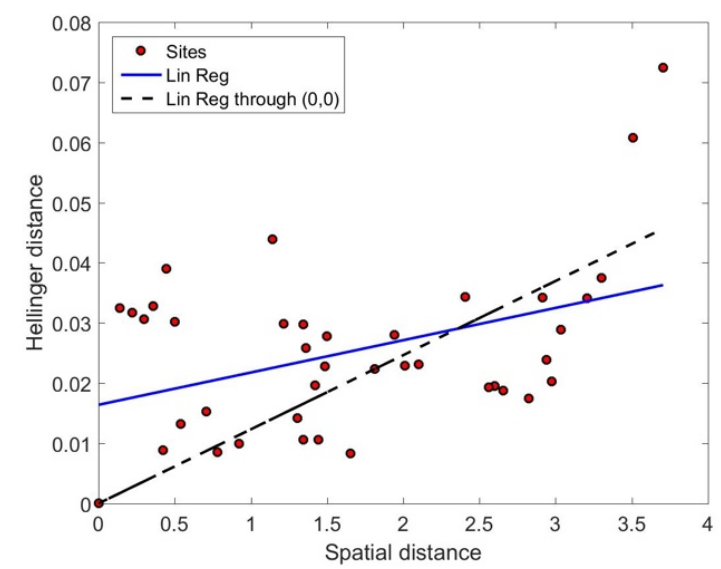

Figure 13: The Hellinger distances (red dots) plotted agains spatial distances for pairs formed by a baseline station and all other units in the cluster. The best linear fit produces statistically significant increasing slope ( $\mathrm{p}$-value $=0.0066)$.

\section{Conclusion}

PV systems observed over hourly intervals appear to have rather uniform outputs. By Markov chain Monte Carlo simulations, we illustrate the effects of short-term intermittency of transient cloudiness and weather disturbances in the atmosphere. We model the generation of geographically dispersed PV systems in minute intervals. The results indicate that geographically distributed PV systems operating in one-minute intervals could cope with peaks of a statewide power grid because of the smoothing effect caused by their geographical spread. In other words, the rapid variations in the generation output of geographically dispersed PV systems are canceled out. However, since PV systems observed in short time intervals can suddenly decrease their output, they create an additional need for a spinning reserve, energy storage, or demand side management that can compensate partly for the uncertainty involved in their output. In fact, because of their intermittency, or sudden energy shortages and overages, PV systems can actually increase the fossil fuel consumption of faster dispatchable spinning reserves, which typically burn the most expensive fuel. Thus, the smooth integration of renewables into microgrids, statewide, and nationwide power grids necessitates their further investigation as a source of supplementary energy, especially for peak power and spinning reserves, and changes in the costs of generation resulting from their intermittency [7]. One possible generalization to be considered in future research is to distinguish Markov chain transition matrices for convective weather disturbances (characteristic for summer and requiring a short memory transition probabilities) and frontal disturbances (more frequent in Fall, Winter, and Spring) in which the Markov chain transition probabilities require longer history.

In order to approach the study of weather induced intermittencies, we propose a few additional analytic tools. The introduced measures of dissimilarity between production patterns show promising applicability as one of the criteria for optimal allocation of solar station clusters/systems by placing PV plants at positions that maximize diversity of the production patterns.

We also plan to propose a wavelet-based optimization procedure where nominal powers of $N$ stations on fixed locations are optimized for nominal distribution of powers, under the constraint that a single station can receive nominal power between 0 and $\mathrm{m}$ MW (maybe discretized).

We generalize the VR coefficient in [20] to total wavelet-based variance reduction (TWVR), and use the maximum TWVR as a goal in optimization procedure. This coefficient is defined as

$$
T V W R=\sum_{\text {year }} \frac{\sum_{i=1}^{N} \operatorname{Var}\left(d_{i}^{*}\right)}{\operatorname{Var}\left(\sum_{i=1}^{N} d_{i}^{*}\right)}
$$

where the $d_{i}^{*}$ s are wavelet coefficients at the finest level of detail representing the intensity of the short term fluctuations in daily output of the $i$ th station summed over a year. In the data set, we analyze the Nyquist time scale of $10^{\prime}$ as the finest wavelet resolution. Intuitively, the above ratio quantifies the capability of a system to cancel the effects of short term fluctuations. One benefit over the Fourier methodology is a time/scale nature of wavelet decompositions, which enables the TWVR coefficient to be defined for arbitrary time intervals during the day, say only for the afternoon output, or only for outputs between 9:00 am and 1:00 pm.

The wavelet based covariances can identify the time scales at which the signals show similarity. In future work, we plan to analyze level-wise covariance matrices of all patterns within a cluster and propose a design that minimizes level-wise "correlation numbers." Such 
problems are common in antenna spatial distribution design where allocations are made to minimize the overall correlation between the units measured via a trace of square of covariance matrix. With this objective function, we can employ an optimization algorithm to find optimal spatial distribution within the constraints imposed by allowable locations.

The purpose of the exercise presented in this paper is to create an analytical framework for integration and optimization of the various generation sources in order to meet the uncertainties of the fast changing aggregate PV output of multiple systems under certain weather conditions, and mitigate its variations through combined use of storage technologies, generation spinning reserve, as well as voluntary and/or involuntary demand response.

\section{Acknowledgement}

The authors gratefully acknowledge financial support of the National Science Foundation under grant \#0836046 (NSF EFRI-RESIN: Sustainable Infrastructures for Energy and Water Supply) which was used for part of the work presented herein. This work was also partially supported by Brook Byers Institute for Sustainable Systems.

\section{References}

[1] J. Bebic, "Power System Planning: Emerging Practices Suitable for Evaluating the Impact of High-Penetration Photovoltaics," NREL/SR-581-42297 Subcontract Report, Feb. 2008.

[2] "Western Wind and Solar Integration Study," NREL/SR-55047434 Subcontract Report, prepared by GE Energy, May 2010.

[3] R. J. Aguiar, M. Collares-Pereira, and J.P. Conde, "Simple procedure for generating sequences of daily radiation values using a library of Markov transition matrices," Solar Energy, vol. 40, pp. 269-279, 1988.

[4] G. Papaefthymiou and B. Klock1, "MCMC for Wind Power Simulation," IEEE Transactions on Energy Conversion, vol. 23, pp. 234-240, 2008.

[5] D. A. McCracken, "Synthetic High Resolution Solar Data," Mater Thesis, Dept. Mechanical Engineering, University of Strathclyde, 2011.
[6] R. Goić, J. Krstulović, and D. Jakus, "Simulation of aggregate wind farm short-term production variations," Renewable Energy, vol. 35, pp. 2602-2609, 2010.

[7] Georgia Power Company, "2010 Annual Report," Tech. Rep., February 25, 2011.

[8] I. Kim, "Impact of Stochastic Renewable Distributed Generation on Urban Distribution Networks," Ph.D. dissertation, Dept. Electrical and Computer Eng., Georgia Institute of Technology, Atlanta, 2014.

[9] United States Census Bureau. Available at the web address: www.census.gov/popest/data/cities/totals/2011/files/SUBEST2011_13.csv

[10] NREL, National Solar Radiation Data Base. Available from: http://rredc.nrel.gov/solar/old_data/nsrdb/1991-2005/tmy3/.

[11] I. Kim, M. Begovic, H. Jeong, and J. Crittenden, "Impact of Photovoltaic Distributed Generation on Generation Resource Allocation," 46th Hawaii International Conference on System Sciences, Wailea, Maui, USA, 7-10 Jan., 2013.

[12] C. Mustacchi, V. Cena, and M. Rocchi, "Stochastic simulation of hourly global radiation sequences," Solar Energy, vol. 23, pp. 47$51,1979$.

[13] R. Bhat, M. Begovic, I. Kim, and J. Crittenden, "Effects of PV on Conventional Generation," 47th Hawaii International Conference on System Sciences, Waikoloa, HI, USA, 6-9 Jan., 2014, pp. 23802387.

[14] NREL, Measurement and Instrumentation Data Center (MIDC). Available from: http://www.nrel.gov/mide/

[15] PVPMC, PV_LIB Toolbox. Available from: http://pvpmc.org/pvlib/

[16] Whitcher, B., Guttorp, P., and Percival, D., "Wavelet analysis of covariance with application to atmospheric time series," Journal of Geophysical Research, 105, D11, DOI: 10.1029/2000JD900110 14941-14962., 2000.

[17] Mallat, S., A Wavelet Tour of Signal Processing, 3rd Edition. The Sparse Way, Academic Press, 2008.

[18] Vidakovic, B.. Statistical Modeling by Wavelets, Wiley, 1999.

[19] Pollard, David E., "A user's guide to measure theoretic probability," Cambridge, UK: Cambridge University Press. ISBN 0-521-002893, 2002 .

[20] M. Lave, J. Kleissl, and J. S. Stein. "A Wavelet-Based Variability Model (WVM) for Solar PV Power Plants," IEEE Transactions on Sustainable Energy, vol. 4(2), pp. 501-509, April 2013. 